\title{
Effects of Weight Loss on Glutathione Peroxidase 3 Serum Concentrations and Adipose Tissue Expression in Human Obesity
}

\author{
Julia Langhardt ${ }^{a} \quad$ Gesine Flehmig $^{a} \quad$ Nora Klöting ${ }^{b}$ Stefanie Lehmann ${ }^{c}$ \\ Thomas Ebert $^{a} \quad$ Matthias Kern ${ }^{a}$ Michael R. Schön ${ }^{d}$ Daniel Gärtner ${ }^{d}$ \\ Tobias Lohmann $^{\mathrm{e}}$ Miriam Dressler ${ }^{\mathrm{e}}$ Mathias Fasshauer ${ }^{\mathrm{a}}$ Peter Kovacs $^{\mathrm{c}}$ \\ Michael Stumvoll ${ }^{a}$ Arne Dietrich ${ }^{f}$ Matthias Blüher ${ }^{a}$ \\ a'Department of Medicine, University of Leipzig, Leipzig, Germany; ${ }^{b}$ IFB ObesityDiseases, \\ Junior Research Group Animal Models, University of Leipzig, Leipzig, Germany; ' $\mathrm{IFB}$

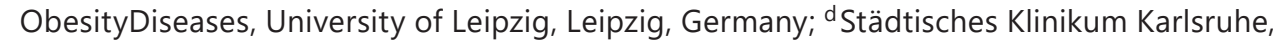

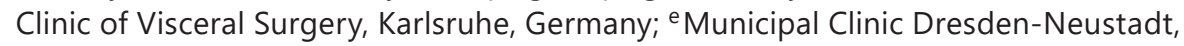

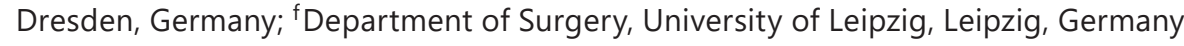

\section{Keywords}

Glutathione peroxidase $3(\mathrm{GPX} 3) \cdot$ Obesity Adipose tissue $\cdot$ Insulin resistance · Adipokines

\begin{abstract}
Background/Aims: Altered expression and circulating levels of glutathione peroxidase 3 (GPX3) have been observed in obesity and type 2 diabetes (T2D) across species. Here, we investigate whether GPX3 serum concentrations and adipose tissue (AT) GPX3 mRNA expression are related to obesity and weight loss. Methods: GPX3 serum concentration was measured in 630 individuals, including a subgroup $(n=293)$ for which omental and subcutaneous (SC) GPX3 mRNA expression has been analyzed. GPX3 analyses include three interventions: 6 months after bariatric surgery $(n=80)$ or combined exercise/hypocaloric diet $(n=20)$ or twostep bariatric surgery $(n=24)$ studies. Results: Bariatric surgery-induced weight loss $(-25.8 \pm$ $8.4 \%)$, but not a moderate weight reduction of $-8.8 \pm 6.5 \%$ was associated with significantly reduced GPX3 serum concentrations. GPX3 mRNA is significantly higher expressed in AT from individuals with normal glucose metabolism compared to T2D patients. SC AT GPX3 expression is significantly higher in lean compared to obese as well as in insulin-sensitive compared insulin-resistant individuals with obesity. Weight loss after bariatric surgery causes a significant increase in SC AT GPX3 expression. AT GPX3 expression significantly correlates with age, $\mathrm{BMI}$, fat distribution, insulin sensitivity (only SC AT), but not with circulating GPX3. Conclusion: Our data support the notion that SC AT GPX3 expression is associated with obesity, fat distribution and related to whole body insulin resistance.

(C) 2018 The Author(s)

Published by S. Karger GmbH, Freiburg
\end{abstract}


Langhardt et al.: Glutathione Peroxidase 3 in Human Obesity

\section{Introduction}

Increased oxidative stress, a state of temporarily or chronically elevated reactive oxygen species (ROS) production, belongs to the main manifestations of obesity and type 2 diabetes (T2D) $[1,2]$. Elevated ROS production may impair cellular metabolism and damage cellular components [3]. Therefore, in healthy cells, there are antioxidant defense mechanisms to detoxify these highly reactive molecules and to maintain low degrees of oxidative stress [2, 4]. Antioxidant enzymes such as superoxide dismutase, catalase, and glutathione peroxidase (GPx) as well as endogenous metabolites are responsible for scavenging and breaking down ROS to less or nonreactive products [2, 4]. At least seven isoforms of GPx proteins have been identified of which glutathione peroxidase 3 (GPX3) accounts for the major anti-oxidative activity in the circulation $[5,6]$.

GPX3 is abundantly expressed in kidney and lung and to a lesser amount in adipose tissue (AT) $[6,7]$. Expression of GPX3 is regulated by hypermethylation and glucocorticoid receptormediated mechanisms [8]. In addition, GPX3 is a direct estrogen receptor alpha target gene in white AT [9]. GPX3 acts as a modulator of redox signaling, has immunomodulatory function, catalyzes the detoxification of ROS, and has been identified as a tumor suppressor in different types of cancer $[6,8]$. Oxidative stress contributes to AT dysfunction in obesity $[10,11]$, hence GPX3 may play a crucial role as local defense mechanism, particularly in obesity $[5,12]$.

Indeed, GPX3 serum concentrations and AT expression have been shown to be dysregulated in human obesity and metabolic disorders $[5,12,13]$ as well as in animal models of obesity and diabetes [5]. In the context of the Diabetes Genome Anatomy Project, GPX3 has been identified as one of the strongest associated genes for the traits insulin sensitivity, adipogenesis, and T2D [14]. We recently found that circulating GPX3 belongs to a cluster of adipokines which is closely related to insulin sensitivity, hyperglycemia, and lipid metabolism [15]. Moreover, the GPX3 gene variant rs8177409 has been shown to be associated with cardiovascular risk in a Mexican population [13]. However, alterations of GPX3 serum concentrations found in association with obesity are heterogeneous and range from significantly increased GPX3 serum concentrations in patients with obesity from Central Mexico [12,13] to markedly decreased plasma GPX3 in a cohort from Korea [5].

Collectively, human and rodent data indicate a role of GPX3 in the maintenance of AT function and systemic defense against increased ROS production and suggest that dysregulation of GPX3 contribute to the link between increased AT mass and obesity-related metabolic and cardiovascular diseases. Here, we sought to determine potential associations between circulating GPX3, AT GPX3 expression, obesity and T2D in a Caucasian cohort with a wide range of BMI, fat distribution, and glucose metabolism parameters. We further tested the hypothesis that weight loss interventions cause changes in GPX3 serum concentrations and AT expression.

\section{Material and Methods}

Subjects

We included six different cohorts with a total number of 630 individuals in our study of GPX3 serum concentration and AT mRNA expression. In the first cohort ( $n=213)$, we investigated GPX3 serum concentrations in relation to measures of obesity and glucose metabolism in a cross-sectional study (Cohort 1) (Table 1). In another cross-sectional study (Cohort 2), we investigated GPX3 mRNA expression in paired visceral omental (vis) and subcutaneous (SC) AT samples in addition to GPX3 serum concentrations $(n=233)$ (Table 2). In addition, 60 morbidly obese men and women with a BMI of $45 \pm 1.3 \mathrm{~kg} / \mathrm{m}^{2}$ scheduled for elective cholecystectomy, explorative laparotomy, or gastric sleeve resection were selected and distributed into two experimental groups of insulin-sensitive (IS) and insulin-resistant (IR) obesity with 30 subjects each as described previously (Cohort 3) [16]. 


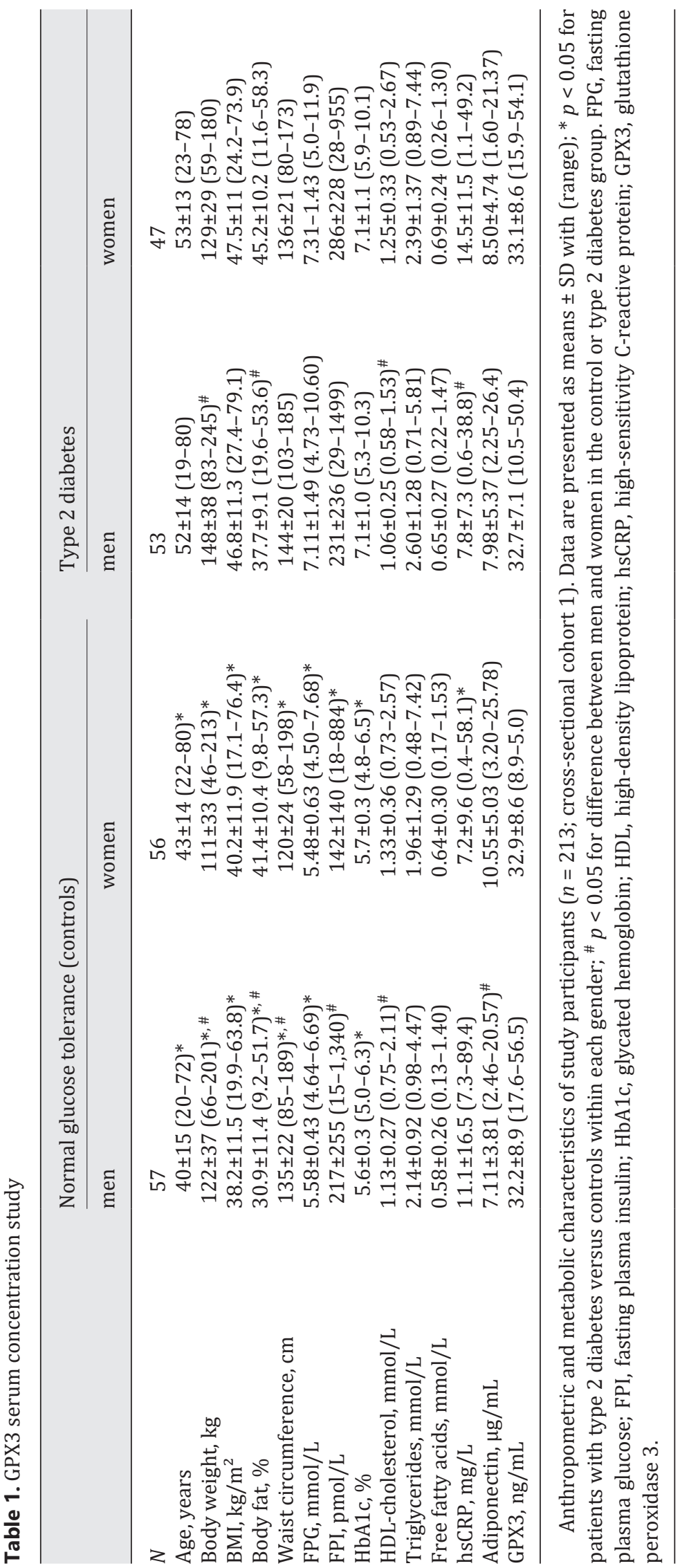




\section{눙}

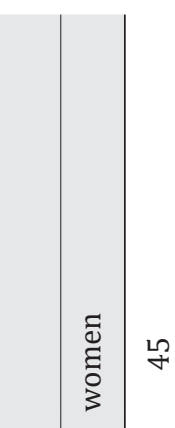

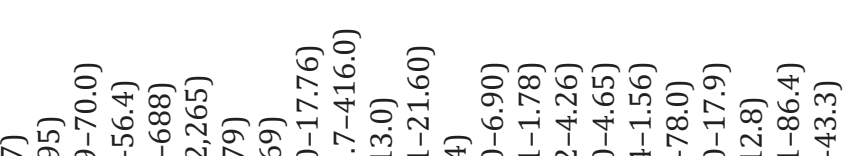

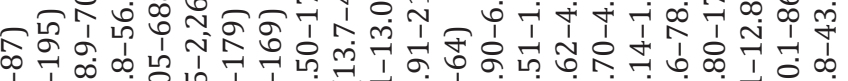

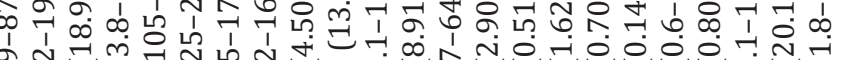

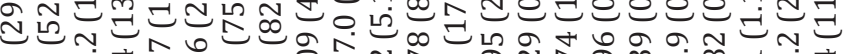
ҰN

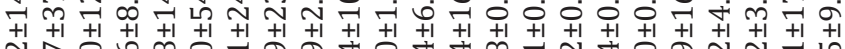
ஸि

бक

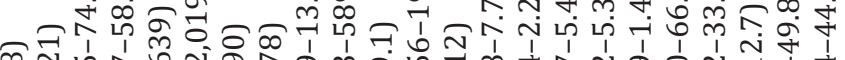
ฉㅊำ 1 m

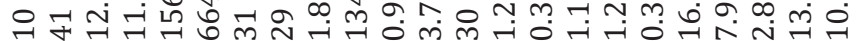

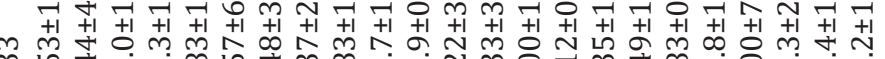

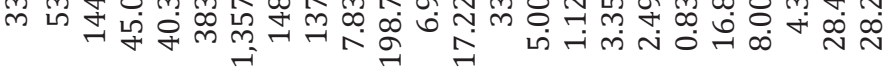

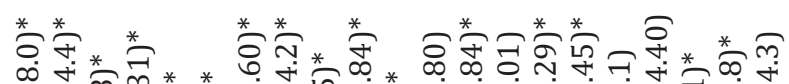

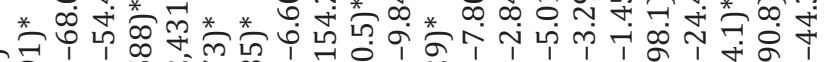

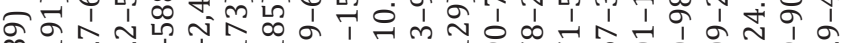
o r + +1 b

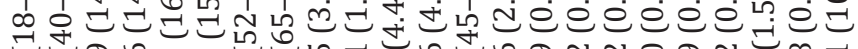

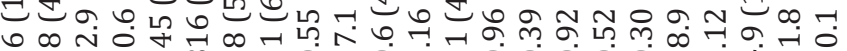

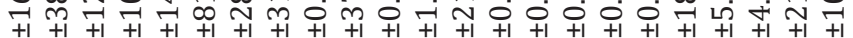
ᄂ

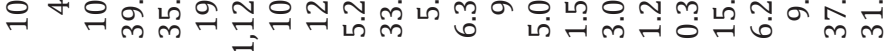

$$
\text { (1) }
$$

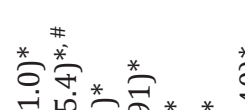

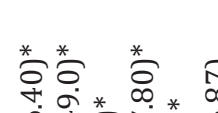

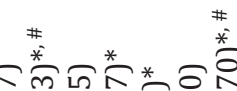
$\stackrel{*^{\circ}}{*}$ *

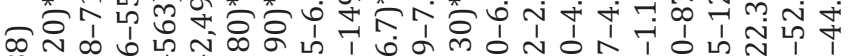
$\infty$ 실

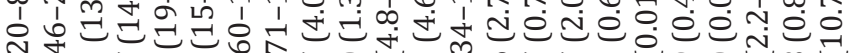
ป⿰讠寸

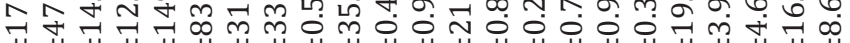

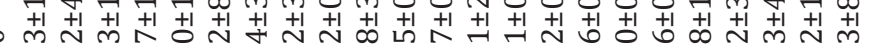
以

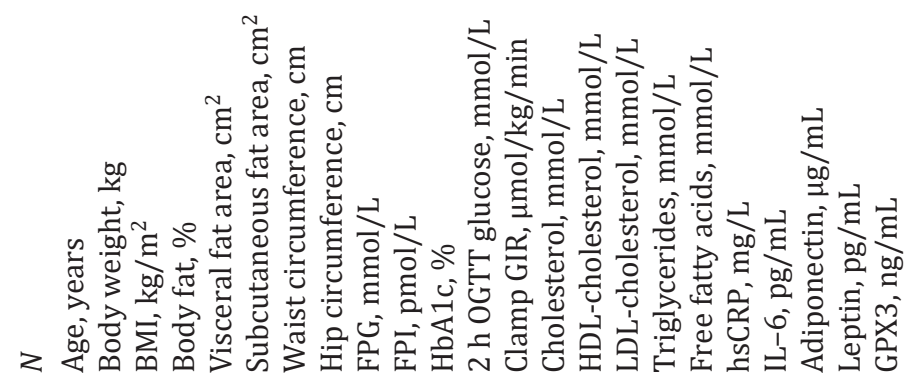


Langhardt et al.: Glutathione Peroxidase 3 in Human Obesity

In interventional studies, we measured circulating GPX3 before and 6 months after a combined exercise and calorie-restricted diet study (Cohort $4, n=20$ ), before and 6 months after bariatric surgery (Cohort 5 , $n=80$ ) (Table 3 ) and before and $12 \pm 2$ months after bariatric surgery in the context of a two-step surgery approach (Cohort 6, $n=24$ ). We included the latter intervention to measure AT GPX3 expression before and after significant weight loss in both visceral and SC fat depots.

We defined the following exclusion criteria: i) Thyroid dysfunction, ii) alcohol or drug abuse, iii) pregnancy, iv) treatment with thiazolidinediones. All studies were approved by the ethics committee of the University of Leipzig (approval numbers: 159-12-21052012 and 017-12-23012012), and all subjects gave written informed consent before taking part in the study.

The cohorts had the following specific characteristics:

Cohort 1

213 Caucasian men $(n=110)$ and women $(n=103)$ have been consecutively recruited in the context of a study on insulin resistance at the Department of Medicine, University of Leipzig, to represent a wide range of obesity, insulin sensitivity, and glucose tolerance. The age ranged from 19 to 80 years and BMI from 17.1 to $79.1 \mathrm{~kg} / \mathrm{m}^{2}$. The study included 100 patients with T2D and 113 normal glucose-tolerant controls (Table 1).

Cohort 2

AT GPX3 mRNA expression was investigated in 233 donors of paired vis and SC AT samples, who underwent abdominal surgery for cholecystectomy, weight reduction surgery, abdominal injuries, or explorative laparotomy (Table 2). From these individuals, 78 had T2D. All subjects had a stable weight, defined as the absence of fluctuations of $>2 \%$ of body weight for at least 3 months before surgery. AT was immediately frozen in liquid nitrogen after explantation. Histologic analyses and measurement of macrophage count in AT was performed as previously described $[16,17]$. To determine adipocyte size, in a subgroup of 122 representative AT donors, $200 \mu \mathrm{L}$ aliquots of adipocytes from both SC and visceral AT were fixed with osmic acid, incubated for $48 \mathrm{~h}$ at $37^{\circ} \mathrm{C}$, and counted in a Coulter counter (Multisizer III; Beckman Coulter, Krefeld, Germany).

\section{Cohort 3}

Insulin-sensitive (IS) and insulin-resistant (IR) obesity study. On the basis of the glucose infusion rate (GIR) in euglycemic hyperinsulinemic clamp, patients were defined as either IS (GIR > $70 \mu \mathrm{mol} / \mathrm{kg} / \mathrm{min}$ ) or IR (GIR < $60 \mu \mathrm{mol} / \mathrm{kg} / \mathrm{min}$ ). Both groups were matched for gender, age, and BMI. Clinical parameters were assessed as described previously [16].

Cohort 4

6 Months Combined Exercise Hypocaloric Diet Study. 20 Caucasian obese volunteers (12 women, 8 men) attending the Obesity Outpatients Clinic at the University of Leipzig Medical Department were recruited (Table 3). Patients underwent a clinical assessment including medical history, physical examination, bioimpedance (BIA) analysis, co-morbidity evaluation as well as nutritional interviews performed by a multidisciplinary consultation team. Weight loss was achieved over a period of 6 months by a diet providing a daily energy deficit of $1,200 \mathrm{kcal} / \mathrm{d}$. Diet adherence was monitored by daily food intake protocols. In addition, study participants were enrolled in $60 \mathrm{~min}$ of supervised physical training sessions two days/week. In brief, each training sessions included 20 min of biking or running, 20 min of swimming, and 20 min of warming up/cooling down periods. All subjects completed a graded bicycle-ergometer test to volitional exhaustion and had maximal oxygen uptake measured with an automated open circuit gas analysis system at baseline. The highest oxygen uptake/min reached was defined as the maximal oxygen uptake, and subjects subsequently trained at their individual submaximal heart rate defined as 70-80\% of the individual maximal heart rate during the bicycle-ergometer test. At baseline as well as after 1, 3 and 6 months, blood samples were obtained in the fasting state, and measurements of anthropometric parameters were performed.

Cohort 5

Bariatric Surgery Study. 80 Caucasian obese volunteers ( 55 women, 25 men) participated in a prospective weight loss study before and 12 months after gastric sleeve resection or Roux-en-Y gastric bypass (Table 3). 


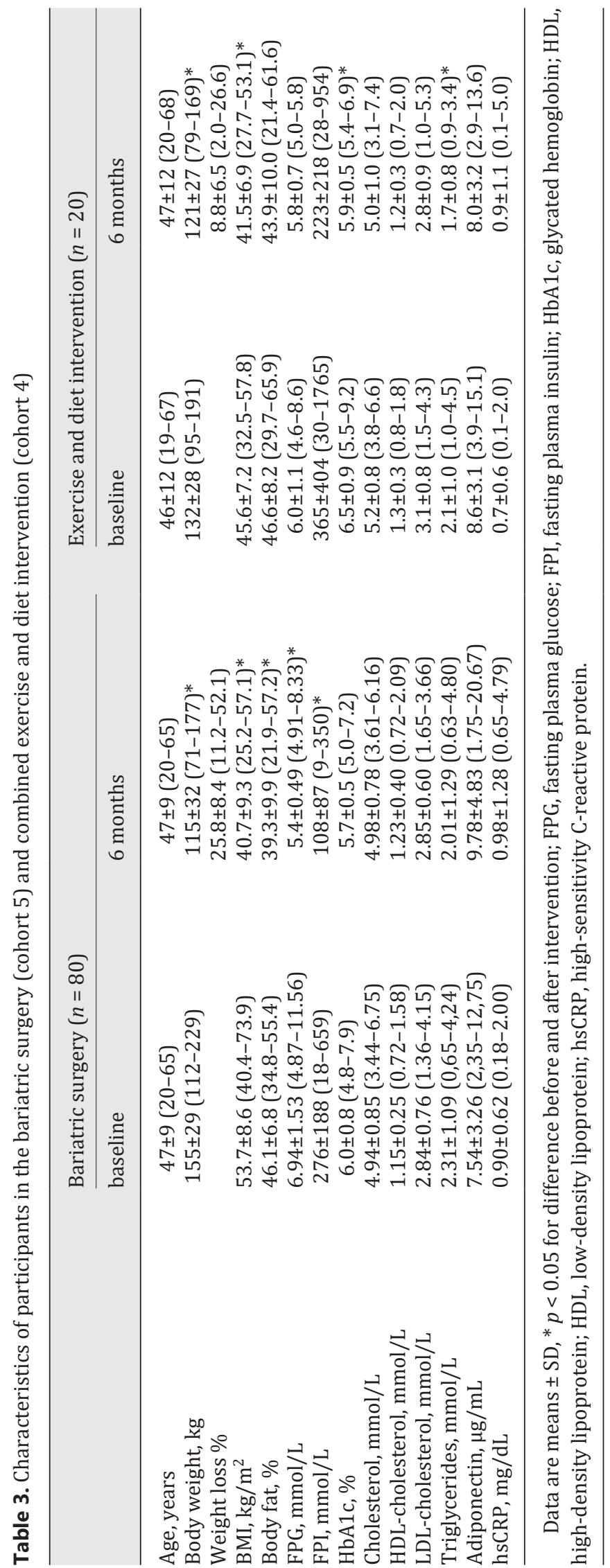


Langhardt et al.: Glutathione Peroxidase 3 in Human Obesity

Cohort 6

Two-Step Bariatric Surgery. In 24 (17 women, 7 men) patients, vis and SC AT biopsies were obtained in the context of a two-step bariatric surgery strategy with gastric sleeve resection as the first step and a Rouxen-Y-gastric bypass as second step operation as previously described [18]. The baseline BMI in this subgroup was $65.3 \pm 8.2 \mathrm{~kg} / \mathrm{m}^{2}$ and the BMI $12 \pm 2$ months after bariatric surgery was $47.1 \pm 8.5 \mathrm{~kg} / \mathrm{m}^{2}$.

Measurement of Body Fat Content, Glucose Metabolism, Insulin Sensitivity

BMI was calculated as weight divided by squared height. Hip circumference was measured over the buttocks; waist circumference was measured at the midpoint between the lower ribs and iliac crest. Percentage body fat was measured by dualX-ray absorptiometry (DEXA) or BIA. In Cohorts 2 and 3, abdominal visceral and SC fat areas were calculated using computed tomography (CT) scans at the level of L4-L5 ( $n=$ 87). Insulin sensitivity was assessed using the HOMA-IR index or with the euglycemic-hyperinsulinemic clamp method as described previously [19].

Analyses of Blood Samples

All baseline blood samples were collected between 8 and 10 a.m. after an overnight fast. Serum GPX3 was measured by an ELISA (Adipogen, Seoul, South Korea). Plasma insulin was measured with an enzyme immunometric assay for the IMMULITE automated analyzer (Diagnostic Products Corporation, Los Angeles, CA, USA). Serum high-sensitive C-reactive protein, adiponectin, IL-6, and leptin were measured as previously described [16].

GPX3 mRNA Expression Studies

Human GPX3 mRNA expression was measured by quantitative real-time polymerase chain reaction (RT-PCR) in a fluorescent temperature cycler using the TaqMan assay, and fluorescence was detected on an ABI PRISM 7000 sequence detector (Applied Biosystems, Darmstadt, Germany). AT was immediately frozen in liquid nitrogen and stored at $-80^{\circ} \mathrm{C}$. RNA was extracted from adipose tissue by using RNeasy Lipid tissue Mini Kit (Qiagen, Hilden, Germany). Quantity and integrity of RNA were monitored with a NanoVue plus Spectrophotometer (GE Healthcare, Freiburg i.Br., Germany). $1 \mu \mathrm{g}$ total RNA from SC and vis AT (305 ng RNA from adipocytes and stromal vascular fraction) were reverse-transcribed with standard reagents (Life technologies, Darmstadt, Germany). cDNA was then proceeded for TaqMan probe-based quantitative RT-PCR (qPCR) using the QuantStudio 6 Flex Real-Time PCR System (Life technologies). Human GPX3 expression was measured by qPCR using human GPX3 probe (Hs00173566_m1). Fluorescence emissions were monitored after each cycle. Human GPX3 mRNA expression was calculated relative to the mRNA expression of hypoxanthine guanine phosphoribosyltransferase 1 (HPRT1) (Hs01003267_m1).

\section{Statistical Analyses}

Data are shown as means \pm SD unless stated otherwise. Before statistical analysis, non-normally distributed parameters were logarithmically transformed to approximate a normal distribution. The following statistical tests were used: paired and unpaired Student's tests, $\chi^{2}$-test, Spearman's correlation, and Pearson's simple correlation. Linear relationships were assessed by least square regression analysis. Statistical analysis was performed using SPSS version 12.0 (IBM, Armonk, NY, USA). $p$ values $<0.05$ were considered to be statistically significant.

\section{Results}

\section{GPX3 Serum Concentration in Obesity and T2D}

Anthropometric and metabolic characteristics of 213 individuals included in the crosssectional study (Cohort 1) are summarized in Table 1. Circulating GPX3 was not different between men and women with normal glucose tolerance and T2D (Fig. 1A). In addition, we did not find significant differences in GPX3 serum concentrations between individuals of different BMI categories (Fig. 1B). In these 213 patients with a wide range of age, BMI, glucose tolerance and insulin sensitivity, we found significant relationships between circulating GPX3 and creatinine $(r=$ -0.14, $p=0.04)$, C-peptide $(r=-0.151, p=0.041)$, fasting triglycerides 
A

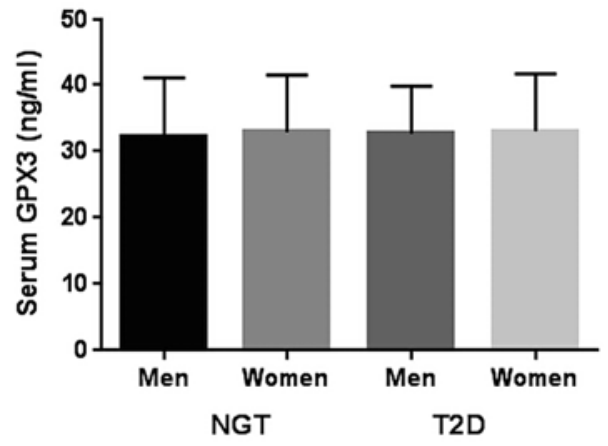

C

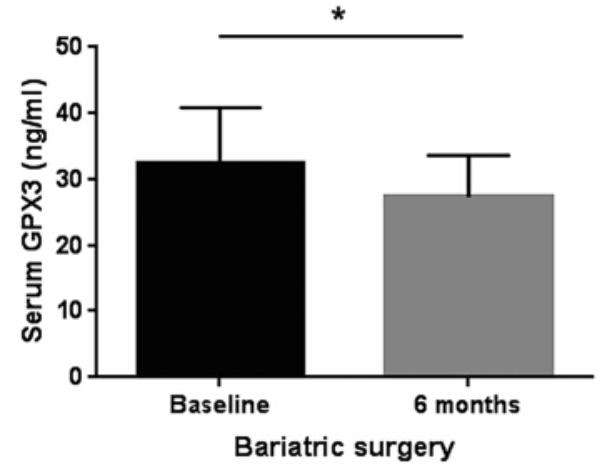

B

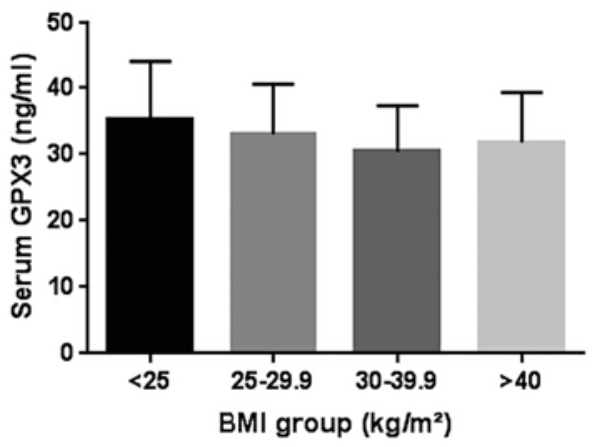

D

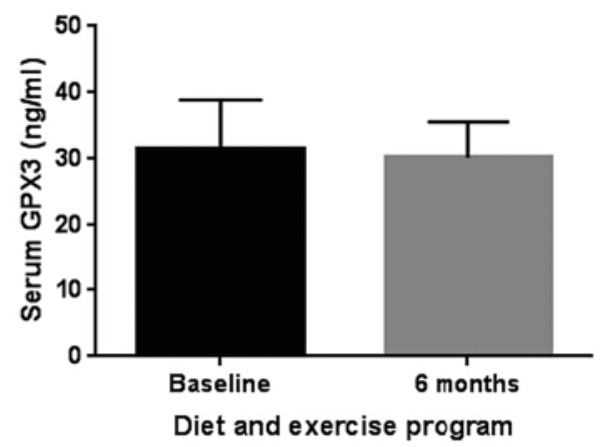

Fig. 1. Glutathione peroxidase 3 (GPX3) serum concentrations in normal glucose-tolerant (NGT) individuals and patients with type 2 diabetes (T2D) and after weight loss interventions. A Circulating GPX3 in men ( $n=$ $57)$ and women $(n=56)$ with NGT and in men $(n=53)$ and women $(n=47)$ with type 2 diabetes (T2D). B GPX3 serum concentrations in lean (BMI $\left.<25 \mathrm{~kg} / \mathrm{m}^{2} ; n=44\right)$, overweight (BMI $\left.>25-29.9 \mathrm{~kg} / \mathrm{m}^{2} ; n=40\right)$, obesity classes I and II (BMI 30-39.9 kg/m²; $\mathrm{n}=65$ ) and obesity class III (BMI $\geq 40 \mathrm{~kg} / \mathrm{m}^{2} ; n=64$ ) NGT individuals. C Effect of Roux-en-Y gastric bypass surgery induced weight loss, 6 months after surgery $(n=80)$ and $\mathbf{D}$ effect of a 6-month combined diet and exercise intervention $(n=20)$ on GPX3 serum concentration. $* p<0.05$ between 6 months after surgery and baseline. Data are means \pm SD.

( $r=-0.162, p=0.023)$, and chemerin $(r=-0.168, p=0.032)$, but not with parameters of (abdominal) obesity, glucose metabolism, insulin sensitivity, and AT biology (data not shown). However, these univariate correlations did not withstand adjustments for age, gender, and BMI.

\section{Changes in GPX3 Serum Concentrations in Response to Different Weight Loss} Interventions

Serum GPX3 concentrations are significantly reduced 6 months after bariatric surgery (Cohort 5, Fig. 1C). Multivariate linear regression analyses identified changes in body weight 
(or BMI) ( $\beta$ coefficient: $0.37, p<0.01$ ) and fasting plasma insulin (FPI) ( $\beta$ coefficient: $0.27, p$ $<0.01$ ) as significant predictors of changes in circulating GPX3.

We further sought to dissect the effects of weight loss and/or improved insulin sensitivity on reduced GPX3 serum concentrations in the context of a 6-month combined exercise hypocaloric diet intervention (Cohort 4). In contrast to the significant effects of bariatric surgery-induced weight loss $(-25.8 \pm 8.4 \%)$, more moderate weight reduction $(-8.8 \pm 6.5 \%)$ and improvements in insulin sensitivity upon this conservative obesity treatment strategy was not sufficient to cause significant changes in GPX3 serum concentrations (Fig. 1D).

\section{GPX3 mRNA Expression in vis and SC AT}

We investigated GPX3 mRNA expression in visceral and SC AT tissue in parallel with GPX3 serum concentrations in 233 individuals, which have been further classified into normal glucose-tolerant (NGT) and T2D patients (Cohort 2, Table 2). Compared to T2D patients, individuals with NGT have significantly higher GPX3 expression both in visceral and SC AT (Fig. 2A). Moreover, there is a trend for higher GPX3 expression in SC compared to visceral fat only in individuals with NGT $(p=0.08)$. This fat depot GPX3 expression difference became statistically significant in lean (BMI $<25 \mathrm{~kg} / \mathrm{m}^{2}$ ) individuals (Fig. 2B). SC GPX3 mRNA expression is significantly higher in AT donors with a BMI $<25 \mathrm{~kg} / \mathrm{m}^{2}$ compared to those with BMI $>30 \mathrm{~kg} / \mathrm{m}^{2}$, whereas no difference in visceral GPX3 expression could be observed between lean and obese individuals (Fig. 2B). There was no difference in both visceral and SC AT GPX3 expression between men and women as well as between premenopausal $(n=45)$ and postmenopausal $(n=105)$ women (data not shown).

To further dissect the potential effects of fat accumulation from those of insulin resistance, we compared GPX3 expression in 60 BMI-, age- and gender-matched patients with either IS $(n=30)$ or IR ( $n=30$ ) obesity (Cohort 3) [16]. Despite a mean BMI of $\sim 45 \mathrm{~kg} / \mathrm{m}^{2}$, we found significantly higher GPX3 mRNA expression in SC compared to vis AT only in IS obesity (Fig. 2C). In addition, SC, but not vis GPX3 mRNA expression was significantly higher in the IS compared to IR obesity subgroup (Fig. 2C). In a prospective bariatric surgery study (Cohort 6), in which we had access to vis and SC AT due to a two-step surgery approach, we confirmed that obesity-related reduced GPX3 expression only affects SC AT (Fig. 2D). Despite a significant weight loss of $\sim 50 \mathrm{~kg}, 12$ months after the first surgical procedure (sleeve gastrectomy), only SC GPX3 expression increased significantly (Fig. 2D). Importantly, circulating GPX3 did not reflect these changes in AT GPX3 expression (GPX3 serum concentration baseline $31.5 \pm$ $6.9 \mathrm{ng} / \mathrm{mL}$ vs. 12 months post-surgery $29.1 \pm 8.4 \mathrm{ng} / \mathrm{mL} ; p=0.4$ ).

We found significant correlations of SC GPX3 mRNA expression with age, BMI, SC and visceral fat areas, mean SC adipocyte size, fasting plasma insulin, and HOMA-IR (Table 4). Vis GPX3 mRNA expression also correlates with age, BMI, mean visceral adipocyte size, SC, and visceral fat areas (Table 4). However, none of these correlations remained significant after adjusting for age, gender, and BMI (Table 4).

\section{Discussion}

Expansion of AT during weight gain is frequently associated with increasingly "stressful conditions" including hypoxia [20], cellular stresses such as oxidative and endoplasmatic reticulum stress [10], autophagy [21], and apoptosis [22], which are reflected by increased macrophage infiltration into AT $[23,24]$. AT dysfunction is associated with local inflammation and ROS accumulation subsequently leading to altered tissue crosstalk through adipokines and AT-derived metabolites [10, 25]. Resulting systemic oxidative stress may link impaired AT function to metabolic abnormalities and cardiometabolic diseases [25]. At the tissue and 
A

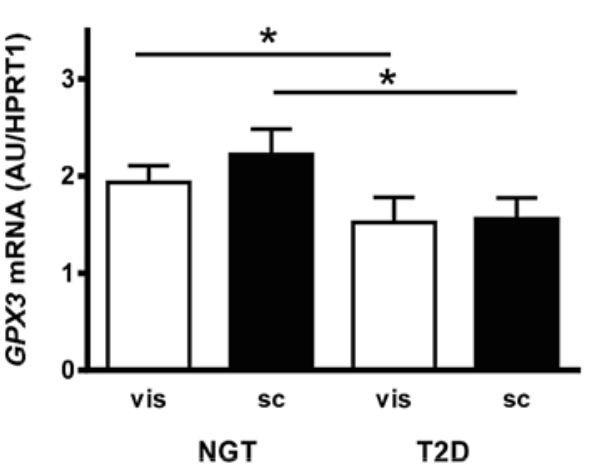

C

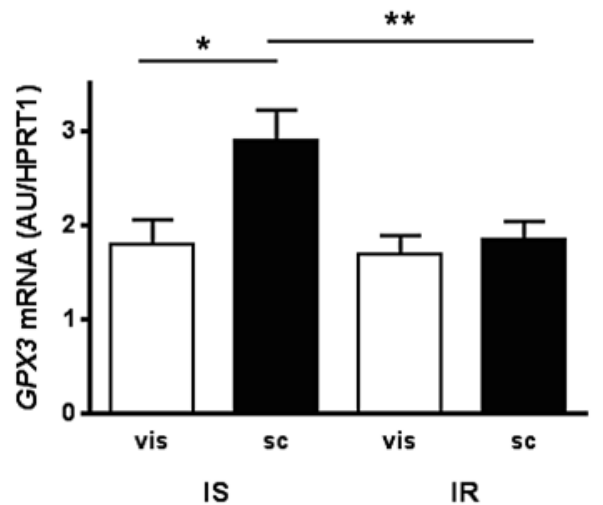

B

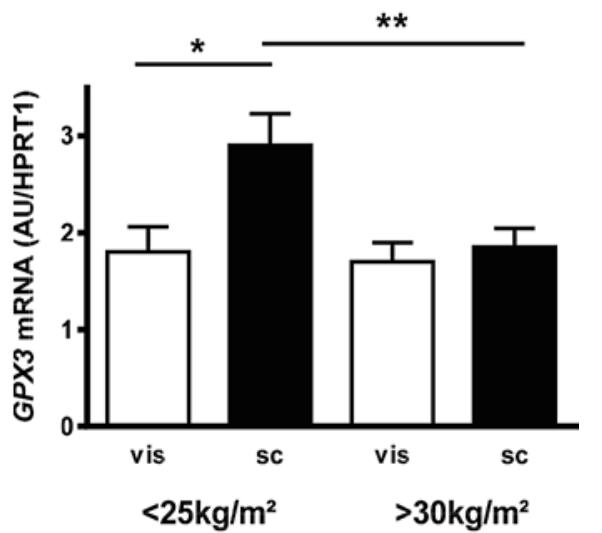

D

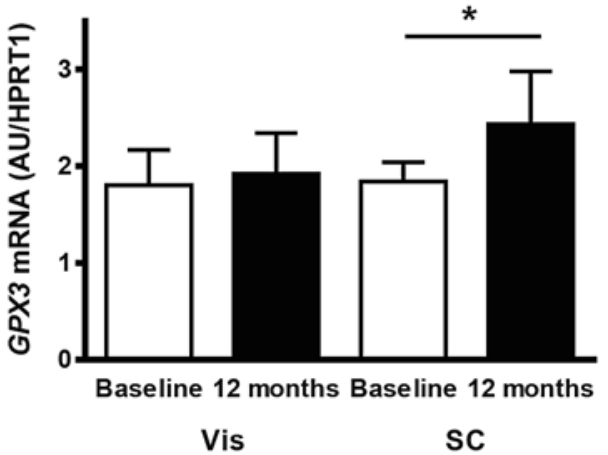

Fig. 2. Glutathione peroxidase 3 (GPX3) mRNA expression in human visceral omental (vis) and subcutaneous (SC) adipose tissue. A GPX3 mRNA expression in the two different fat depots in normal glucose-tolerant (NGT) individuals $(n=155)$ and patients with type 2 diabetes (T2D) $(n=78)$. B Vis and SC GPX3 mRNA expression in lean (BMI $\left.<25 \mathrm{~kg} / \mathrm{m}^{2}, n=25\right)$ and obese (BMI $\left.>30 \mathrm{~kg} / \mathrm{m}^{2}, n=190\right)$ individuals. C GPX3 mRNA expression in vis und SC adipose tissue of individuals with either insulin-sensitive (IS, $n=30)$ or insulin-resistant (IR, $n=$ 30 ) obesity. On the basis of the glucose infusion rate (GIR) in euglycemic-hyperinsulinemic clamps, patients were defined as either IS (GIR $>70 \mu \mathrm{mol} / \mathrm{kg} / \mathrm{min}$ ) or IR (GIR $<60 \mu \mathrm{mol} / \mathrm{kg} / \mathrm{min}$ ). Both groups were matched for gender, age, and BMI. D GPX3 mRNA expression in vis and SC adipose tissue after significant $48 \pm 12 \mathrm{~kg}$ weight loss in response to bariatric surgery. 24 patients underwent a two-step bariatric surgery strategy with gastric sleeve resection as the first step and a Roux-en-Y gastric bypass as second step $12 \pm 2$ months later. At both time points, vis and SC adipose tissue were obtained. ${ }^{* *} p<0.01,{ }^{*} p<0.05$. Data are means \pm SEM.

systemic level, there are defense mechanisms against increased ROS production which include antioxidant enzymes such as superoxide dismutase, catalase, and GPX [2, 4]. There are several lines of evidence suggesting that conditions promoting oxidative stress (e.g. hypoxia, inflammation) may also cause down-regulation of GPX3 expression in AT and in the 
Langhardt et al.: Glutathione Peroxidase 3 in Human Obesity

Table 4. Correlation analyses of GPX3 mRNA expression in visceral and subcutaneous adipose tissue with parameters of obesity, fat distribution and insulin sensitivity in cohort $2(n=233)$

\begin{tabular}{|c|c|c|c|c|}
\hline & \multicolumn{2}{|c|}{ Subcutaneous GPX3 mRNA } & \multicolumn{2}{|l|}{ Visceral GPX3 mRNA } \\
\hline & $\begin{array}{l}r \\
\text { (BMI, age and sex } \\
\text { adjusted) }\end{array}$ & $\begin{array}{l}p \text { value } \\
\text { (BMI, age and sex } \\
\text { adjusted) }\end{array}$ & $\begin{array}{l}r \\
\text { (BMI, age and sex } \\
\text { adjusted) }\end{array}$ & $\begin{array}{l}p \text { value } \\
\text { (BMI, age and } \\
\text { sex adjusted) }\end{array}$ \\
\hline Age, years & 0.168 & 0.010 & 0.146 & 0.026 \\
\hline BMI, kg & -0.260 & $<0.0001$ & -0.143 & 0.029 \\
\hline Subcutaneous fat area, $\mathrm{cm}^{2}(n=87)$ & $-0.270(-0.09)$ & $<0.0001(0.20)$ & $-0.162(-0.03)$ & $0.022(0.47)$ \\
\hline Mean subcutaneous adipocyte size $(\mathrm{pl})(n=120)$ & $-0.311(-0.1)$ & $<0.0001(0.14)$ & $-0.137(0.01)$ & $0.034(0.62)$ \\
\hline Visceral fat area, $\mathrm{cm}^{2}(n=87)$ & $-0.227(-0.07)$ & $0.001(0.28)$ & $-0.146(-0.04)$ & $0.039(0.32)$ \\
\hline Mean visceral adipocyte size, $\mathrm{pl}(n=120)$ & $-0.175(-0.02)$ & $0.021(0.56)$ & $-0.192(-0.08)$ & $0.008(0.31)$ \\
\hline Fasting plasma insulin, pmol/L & $-0.180(-0.03)$ & $0.016(0.44)$ & $-0.121(-0.02)$ & $0.106(0.52)$ \\
\hline HOMA-IR & $-0.176(-0.03)$ & $0.018(0.43)$ & $-0.126(-0.03)$ & $0.106(0.41)$ \\
\hline
\end{tabular}

$r$, Spearman's correlation coefficient; in brackets: $r$ and $p$ values adjusted for BMI, age, and sex. HOMA-IR, homeostatic model assessment - insulin resistance.

circulation. In this context, decreased plasma GPX3 has been shown in Korean patients with obesity [5], children with increased risk for idiopathic stroke [26], and patients with ovarian cancer [27], ischemic heart disease [28], type 2 diabetes [29] and other diseases. These data are supported by significantly lower GPX3 plasma concentrations observed in animal models for obesity $(o b / o b)$ and type 2 diabetes $(d b / d b)$ [5]. Both in human studies and in animal models, reduced GPX3 plasma concentration and activity could be related to decreased expression of GPX3 in AT [5, 30].

In our study, we did not find differences in GPX3 serum concentrations in patients with obesity compared to lean and overweight individuals or in patients with T2D compared to individuals with normal glucose metabolism. Our data are in contrast to the reported reduced GPX3 plasma activity and concentration in patients with obesity from Korea [5] and the previously shown higher GPX3 serum concentrations in overweight and obese patients from Central Mexico $[12,13]$. Given these heterogeneous observations, it is tempting to speculate that ethnic differences between our Caucasian and the previously investigated $[5,12,13]$ cohorts from other ethnicities contribute to different associations between circulating GPX3 and obesity. Indeed, GPX3 allele diversity has been shown in Brazilian, Caucasian, African, and Asian populations [31]. Although polymorphisms in the GPX3 promoter region could not directly been linked to circulating GPX3, distinct GPX3 gene polymorphisms may contribute to a higher risk of obesity [13], ischemic stroke [31], and cerebral venous thrombosis [13, 31]. In addition, phenotype differences between the human cohorts as well as differences in smoking status, concomitant medication, dietary habits and others may underlie the heterogeneous associations between GPX3, obesity and T2D. In this context, glitazones have been shown to regulate GPX3 expression and GPX3 serum concentrations in mice and man [5, 30]. However, in our study, patients treated with thiazolidinediones have been excluded. An important limitation of our study is that we only report GPX3 serum protein, but not activity. On the other hand, GPX3 protein concentration in the circulation and activity are correlated $[5,32]$, suggesting that we could extrapolate GPX3 activity from GPX3 serum concentrations. Noteworthy, we and others $[5,12,13]$ did not find previously suggested gender differences with $\sim 17 \%$ higher GPX3 serum concentration in women as compared to men [32].

To further elucidate the heterogeneous associations between circulating GPX3 and obesity observed in different cohorts, we analyzed GPX3 serum concentrations in two inde- 
Langhardt et al.: Glutathione Peroxidase 3 in Human Obesity

pendent weight loss interventions. We found significantly reduced GPX3 serum concentrations in morbidly obese patients 6 months after bariatric surgery. The reduction in GPX3 serum concentrations closely corresponds to the approximately $26 \%$ decreased body weight. In contrast, approximately 9\% lower BMI 6 months after a combined hypocaloric diet and exercise program did not cause changes in GPX3 serum concentrations, suggesting that the extent of weight loss may determine changes in circulating GPX3. We could not confirm significantly increased GPX3 serum concentrations observed in previous diet and exercise interventions in patients with a BMI between $31-35 \mathrm{~kg} / \mathrm{m}^{2}$ [33] or $<40 \mathrm{~kg} / \mathrm{m}^{2}$ [34]. Because the majority of individuals included into our weight loss intervention had a BMI $>40 \mathrm{~kg} / \mathrm{m}^{2}$, we suggest that individuals with a lower BMI and presumably earlier stage of obesity with less GPX3-suppressing AT inflammation are still able to activate GPX3 upon diet and exercise interventions.

Our data on reduced GPX3 serum concentrations upon bariatric surgery-induced weight loss may support data from the study in Central Mexico which found significantly higher circulating GPX3 in overweight and obese patients. Discrepant correlations between body weight and circulating GPX3 may be due to differences in the regulation of GPX3 expression in different tissues. Kidney and lung have been considered the main source for circulating GPX3 $[6,7]$. This view has been challenged by data demonstrating that reduced AT but not lung and kidney GPX3 expression translates into reduced GPX3 plasma activity [5]. We therefore systematically studied abdominal visceral and SC AT GPX3 mRNA expression in parallel to GPX3 serum concentrations in a large cohort of individuals with a wide range of obesity, fat distribution and glucose metabolism / insulin sensitivity parameters. In addition, we included analyses of extreme obesity subphenotypes with IS compared to IR obesity [16] and a two-step bariatric surgery approach allowing to investigate changes in GPX3 AT expression (both SC and visceral depots) longitudinally [18, 35].

Indeed, we confirmed the previously reported reduced GPX3 expression in AT in obesity [5] and T2D. Interestingly, GPX3 mRNA was significantly higher in both visceral and SC fat depots in individuals with normal glucose metabolism as compared to T2D patients, whereas the comparison of AT GPX3 expression in different BMI categories only revealed significantly lower GPX3 expression in SC, but not in vis AT. Because GPX3 is an estrogen receptor alpha target gene in AT [9], we expected gender differences in AT GPX3 expression. Interestingly, we neither found differences between men and women nor between pre- and postmenopausal women in both vis and SC AT GPX3 expression. This suggests that factors other than estrogen may predominantly modulate GPX3 gene expression in human AT. Stress-related factors including ROS may represent more important modulators of GPX3 expression in AT $[5,12]$. Under stressful conditions such as inflammation and hypoxia [36], local AT GPX3 expression is suppressed. However, we did not measure oxidative stress in AT as previous studies did [5] and can therefore not draw any conclusion from our own data that dysregulation of GPX3 may contribute to adipocyte and AT dysfunction.

In SC AT, we found significant negative correlations between GPX3 expression and BMI, abdominal SC and visceral fat areas, adipocyte size as well as fasting plasma insulin and HOMA-IR, whereas in visceral AT, we only found GPX3 expression to be correlated with BMI, abdominal SC and visceral fat areas, and mean adipocyte size. Abdominal visceral fat distribution and AT dysfunction predominantly in visceral fat have been linked to insulin resistance $[11,13]$. Therefore, it was surprising that GPX3 expression only in SC AT correlates with parameters of insulin sensitivity. However, associations between markers of insulin sensitivity and GPX3 expression in SC AT were not significant after adjusting for age, gender and BMI, suggesting that GPX3 in AT does independently of possible age and BMI effects contribute to the link between AT dysfunction, insulin resistance and impaired glucose metabolism. In contrast, we found that patients with IS obesity have significantly higher GPX3 in SC AT 
compared to IR but obese (BMI $\sim 45 \mathrm{~kg} / \mathrm{m}^{2}$ ) patients. The fact that we cannot confirm an ageand BMI-independent relationship between AT GPX3 expression and HOMA-IR may be explained by the somewhat artificial model of IS versus IR obesity, which disregards the range of BMI in relation to insulin sensitivity. Based on these data, we conclude that AT GPX3 expression does not play a causative role in the link between AT dysfunction and whole-body insulin resistance. We have previously shown that despite matching for BMI, age, gender and total body fat mass, patients with IS obesity are to some extent protected against visceral and ectopic fat deposition and AT inflammation [16]. Data from this biological model of maintained insulin sensitivity independently of obesity therefore suggest that impaired expandability of SC AT, adipocyte hypertrophy, and AT inflammation may cause reduced GPX3 expression. Moreover, our data support the notion that GPX3 expression in AT could be diminished by local pro-inflammatory factors such as TNF- $\alpha$ and lipopolysaccharide [5]. Low AT inflammation and low expression of pro-inflammatory cytokines are hallmarks of AT biology in patients with IS obesity [16].

Using a two-step bariatric surgery with sleeve gastrectomy as first and, about 12 months later, Roux-en-Y gastric bypass surgery as second step, we were able to evaluate longitudinal GPX3 expression changes in both abdominal visceral and SC AT. Importantly, patients in the two-step bariatric surgery group were not in a negative energy balance at the time of the second surgery, because a stable body weight over at least 3 months was a prerequisite and indication for the second surgical procedure. We found that upon significant weight loss, GPX3 expression significantly increased only in SC AT. These data suggest that SC AT GPX3 is more responsive to weight-loss associated changes in AT than visceral GPX3 expression These data further support the notion that AT GPX3 expression is regulated in a fat depotspecific pattern.

Interestingly, we did not find significant associations between these differences or changes in SC AT GPX3 expression and GPX3 serum concentrations. Paradoxically and in contrast to expectations from previous studies [5], increased SC AT GPX3 expression was associated with lower GPX3 serum concentrations after bariatric surgery-induced weight loss. In cross-sectional analyses, we did not find correlations between AT GPX3 expression (both fat depots) and circulating GPX3. Even in the model of IS versus IR obesity, an approximately 40\% increased SC AT GPX3 expression was not related to differences in GPX3 serum concentrations between these obese subphenotypes.

It has been reported that the release of GPX3 from AT depends on total AT mass, and in vitro GPX3 secretion per kilogram AT decreases with increasing total body fat mass [37]. However, it remains unclear whether and how differences in total body fat mass affect in vivo GPX3 release from AT in humans. Moreover, GPX3 serum concentrations reflect the net (and combined) release from kidney, lung, AT, and other organs. The absence of the relationship between AT GPX3 expression and circulating GPX3 suggests that at least in our cohort, tissues other than AT may determine GPX3 serum concentrations. In obese Otsuka-Long-EvansTokushima Fatty (OLETF) rats, increased serum GPX was due to increased secretion of GPX from the kidney, but not from AT [30]. In contrast, in mouse models for obesity and diabetes, decreased GPX3 serum activity was primarily caused by reduced AT GPX3 expression, whereas kidney GPX3 expression was not related to obesity [5].

In the context of human studies, it is difficult to systematically analyze the contribution of different organs to circulating GPX3. It therefore remains an open question which tissues predominantly underlie the reported associations of circulating GPX3 with obesity $[5,12,13]$, weight loss (own data), cardiovascular diseases [13, 31], T2D [29], insulin sensitivity, hyperglycemia, lipid metabolism [15], and certain types of cancer [27, 38, 39].

In summary, we extent previous findings that obesity is associated with decreased AT GPX3 expression by demonstrating that significant weight loss after bariatric surgery can 
significantly increase SC AT GPX3 expression. Moreover, our data from patients with IS obesity suggest that GPX3 expression in AT is regulated by factors other than increased fat mass. Our human studies support data from a rat model of obesity that AT GPX3 expression does not significantly contribute to altered circulating GPX3 concentrations.

Collectively, our data suggest that dysregulation of GPX3 is associated with obesity and fat distribution and related to whole body insulin resistance. However, our data do not allow to draw conclusions whether AT GPX3 expression may reflect or contribute to AT dysfunction in obesity and IR states.

\section{Acknowledgments}

The authors would like to thank all study participants, Daniela Kern and Susan Berthold for technical assistance.

\section{Funding}

This work was funded by the Deutsche Forschungsgemeinschaft: Collaborative Research Center SFB1052, project B1 (to MB) and supported by the Kompetenznetz Adipositas (Competence network for Obesity) funded by the Federal Ministry of Education and Research (FKZ 01GI0829), the Helmholtz Alliance ICEMED - Imaging and Curing Environmental Metabolic Diseases, through the Initiative and Networking Fund of the Helmholtz Association and the German Center for Diabetes Research (DZD), (grant: 82DZD00601). This work has received support from the EU/EFPIA Innovative Medicines Initiative Joint Undertaking (EMIF grant no 115372).

\section{Disclosure Statement}

The authors do not have any conflict of interest related to this manuscript. We disclose any financial conflict of interest.

\section{References}

1 Matsuda M, Shimomura I. Increased oxidative stress in obesity: implications for metabolic syndrome, diabetes, hypertension, dyslipidemia, atherosclerosis, and cancer. Obes Res Clin Pract. 2013 Sep-0ct;7(5):e330-41.

2 Pesta D, Roden M. The Janus Head of Oxidative Stress in Metabolic Diseases and During Physical Exercise. Curr Diab Rep. 2017 Jun;17(6):41.

3 Lushchak VI. Free radicals, reactive oxygen species, oxidative stress and its classification. Chem Biol Interact. 2014 Dec;224:164-75.

4 Brown DI, Griendling KK. Regulation of signal transduction by reactive oxygen species in the cardiovascular system. Circ Res. 2015 Jan;116(3):531-49.

5 Lee YS, Kim AY, Choi JW, Kim M, Yasue S, Son HJ, et al. Dysregulation of adipose glutathione peroxidase 3 in obesity contributes to local and systemic oxidative stress. Mol Endocrinol. 2008 Sep;22(9):2176-89.

6 Drevet JR. The antioxidant glutathione peroxidase family and spermatozoa: a complex story. Mol Cell Endocrinol. 2006 May;250(1-2):70-9.

7 Takahashi K, Avissar N, Whitin J, Cohen H. Purification and characterization of human plasma glutathione peroxidase: a selenoglycoprotein distinct from the known cellular enzyme. Arch Biochem Biophys. 1987 Aug; 256(2):677-86.

8 An BC, Jung NK, Park CY, Oh IJ, Choi YD, Park JI, Lee SW: Epigenetic and glucocorticoid receptor-mediated regulation of glutathione peroxidase 3 in lung cancer cells. Mol Cells 2016;39:631-8.

9 Lundholm L, Putnik M, Otsuki M, Andersson S, Ohlsson C, Gustafsson JA, et al. Effects of estrogen on gene expression profiles in mouse hypothalamus and white adipose tissue: target genes include glutathione peroxidase 3 and cell death-inducing DNA fragmentation factor, alpha-subunit-like effector A. J Endocrinol. 2008 Mar;196(3):547-57. 
Langhardt et al.: Glutathione Peroxidase 3 in Human Obesity

10 Rudich A, Kanety H, Bashan N. Adipose stress-sensing kinases: linking obesity to malfunction. Trends Endocrinol Metab. 2007 Oct;18(8):291-9.

11 Blüher M. Adipose tissue dysfunction contributes to obesity related metabolic diseases. Best Pract Res Clin Endocrinol Metab. 2013 Apr;27(2):163-77.

12 Baez-Duarte BG, Zamora-Ginez I, Mendoza-Carrera F, Ruiz-Vivanco G, Torres-Rasgado E, Gonzalez-Mejia ME, et al. Serum levels of glutathione peroxidase 3 in overweight and obese subjects from central Mexico. Arch Med Res. 2012 Oct;43(7):541-7.

13 Baez-Duarte BG, Mendoza-Carrera F, García-Zapién A, Flores-Martínez SE, Sánchez-Corona J, Zamora-Ginez I, et al.; Multidisciplinary Research Group on Diabetes of the Instituto Mexicano del Seguro Social. Glutathione peroxidase 3 serum levels and GPX3 gene polymorphisms in subjects with metabolic syndrome. Arch Med Res. 2014 Jul;45(5):375-82.

14 Park PJ, Kong SW, Tebaldi T, Lai WR, Kasif S, Kohane IS. Integration of heterogeneous expression data sets extends the role of the retinol pathway in diabetes and insulin resistance. Bioinformatics. 2009 Dec;25(23): 3121-7.

15 Flehmig G, Scholz M, Klöting N, Fasshauer M, Tönjes A, Stumvoll M, et al. Identification of adipokine clusters related to parameters of fat mass, insulin sensitivity and inflammation. PLoS One. 2014 Jun;9(6):e99785.

16 Klöting N, Fasshauer M, Dietrich A, Kovacs P, Schön MR, Kern M, et al. Insulin-sensitive obesity. Am J Physiol Endocrinol Metab. 2010 Sep;299(3):E506-15.

17 Krist J, Wieder K, Klöting N, Oberbach A, Kralisch S, Wiesner T, et al. Effects of weight loss and exercise on apelin serum concentrations and adipose tissue expression in human obesity. Obes Facts. 2013;6(1):57-69.

18 Schmitz J, Evers N, Awazawa M, Nicholls HT, Brönneke HS, Dietrich A, et al. Obesogenic memory can confer long-term increases in adipose tissue but not liver inflammation and insulin resistance after weight loss. Mol Metab. 2016 Jan;5(5):328-39.

19 Blüher M, Unger R, Rassoul F, Richter V, Paschke R. Relation between glycaemic control, hyperinsulinaemia and plasma concentrations of soluble adhesion molecules in patients with impaired glucose tolerance or Type II diabetes. Diabetologia. 2002 Feb;45(2):210-6.

20 Trayhurn P, Wang B, Wood IS. Hypoxia in adipose tissue: a basis for the dysregulation of tissue function in obesity? Br J Nutr. 2008 Aug; 100(2):227-35.

21 Maixner N, Kovsan J, Harman-Boehm I, Blüher M, Bashan N, Rudich A. Autophagy in adipose tissue. Obes Facts. 2012;5(5):710-21.

22 Wabitsch M. The acquisition of obesity: insights from cellular and genetic research. Proc Nutr Soc. 2000 May; 59(2):325-30

23 Weisberg SP, McCann D, Desai M, Rosenbaum M, Leibel RL, Ferrante AW Jr. Obesity is associated with macrophage accumulation in adipose tissue. J Clin Invest. 2003 Dec;112(12):1796-808.

24 Blüher M. Adipose tissue inflammation: a cause or consequence of obesity-related insulin resistance? Clin Sci (Lond). 2016 Sep;130(18):1603-14.

25 Furukawa S, Fujita T, Shimabukuro M, Iwaki M, Yamada Y, Nakajima Y, et al. Increased oxidative stress in obesity and its impact on metabolic syndrome. J Clin Invest. 2004 Dec;114(12):1752-61.

26 Kenet G, Freedman J, Shenkman B, Regina E, Brok-Simoni F, Holzman F, et al. Plasma glutathione peroxidase deficiency and platelet insensitivity to nitric oxide in children with familial stroke. Arterioscler Thromb Vasc Biol. 1999 Aug;19(8):2017-23.

27 Agnani D, Camacho-Vanegas O, Camacho C, Lele S, Odunsi K, Cohen S, et al. Decreased levels of serum glutathione peroxidase 3 are associated with papillary serous ovarian cancer and disease progression. J Ovarian Res. 2011 Oct;4(1):18.

28 Freedman JE, Frei B, Welch GN, Loscalzo J. Glutathione peroxidase potentiates the inhibition of platelet function by S-nitrosothiols. J Clin Invest. 1995 Jul;96(1):394-400.

29 Wilson KH, Eckenrode SE, Li QZ, Ruan QG, Yang P, Shi JD, et al. Microarray analysis of gene expression in the kidneys of new- and post-onset diabetic NOD mice. Diabetes. 2003 Aug;52(8):2151-9.

30 Asayama K, Nakane T, Dobashi K, Kodera K, Hayashibe H, Uchida N, et al. Effect of obesity and troglitazone on expression of two glutathione peroxidases: cellular and extracellular types in serum, kidney and adipose tissue. Free Radic Res. 2001 Apr;34(4):337-47.

31 Grond-Ginsbach C, Lichy C, Padovani A, Pezzini A. GPx-3 gene promoter variation and the risk of arterial ischemic stroke. Stroke. 2007 Jun;38(6):e23.

32 Rush JW, Sandiford SD. Plasma glutathione peroxidase in healthy young adults: influence of gender and physical activity. Clin Biochem. 2003 Jul;36(5):345-51.

33 Abd El-Kader SM, Saiem Al-Dahr MH. Impact of weight loss on oxidative stress and inflammatory cytokines in obese type 2 diabetic patients. Afr Health Sci. 2016 Sep;16(3):725-33.

34 Bougoulia M, Triantos A, Koliakos G. Plasma interleukin-6 levels, glutathione peroxidase and isoprostane in obese women before and after weight loss. Association with cardiovascular risk factors. Hormones (Athens). 2006 Jul-Sep;5(3):192-9.

35 Mardinoglu A, Heiker JT, Gärtner D, Björnson E, Schön MR, Flehmig G, et al. Extensive weight loss reveals distinct gene expression changes in human subcutaneous and visceral adipose tissue. Sci Rep. 2015 Oct; 5(1): 14841.

36 Bierl C, Voetsch B, Jin RC, Handy DE, Loscalzo J. Determinants of human plasma glutathione peroxidase (GPx-3) expression. J Biol Chem. 2004 Jun;279(26):26839-45. 
Langhardt et al.: Glutathione Peroxidase 3 in Human Obesity

37 Fain JN, Tagele BM, Cheema P, Madan AK, Tichansky DS. Release of 12 adipokines by adipose tissue, nonfat cells, and fat cells from obese women. Obesity (Silver Spring). 2010 May;18(5):890-6.

38 Yu YP, Yu G, Tseng G, Cieply K, Nelson J, Defrances M, et al. Glutathione peroxidase 3, deleted or methylated in prostate cancer, suppresses prostate cancer growth and metastasis. Cancer Res. 2007 Sep;67(17):8043-50.

39 Lee HJ, Do JH, Bae S, Yang S, Zhang X, Lee A, et al. Immunohistochemical evidence for the over-expression of Glutathione peroxidase 3 in clear cell type ovarian adenocarcinoma. Med Oncol. 2011 Dec;28(S1 Suppl 1):S522-7. 